\title{
Research on tracking parameter calibration method of target miss-distance for laser tracker
}

\author{
DONG Deng-feng ${ }^{1, a^{*}}$, CHENG Zhi ${ }^{1, b}$, CUI Cheng-jun ${ }^{1,2, c}$, ZHOU Weihu ${ }^{1, d}$ \\ and JI Rong-yi, ${ }^{1, e}$ \\ ${ }^{1}$ Academy of Opto-electronics, Chiese Academy of Sciences, Beijing 100094, China \\ ${ }^{2}$ Changchun University of Science and Technology, changchun 130022, China \\ adongdengfeng@aoe.ac.cn, ${ }^{b} 15010680651 @ 163 . c o m,{ }^{e}$ ccj408@126.com, \\ dzhouweihu@aoe.ac.cn, ${ }^{\mathrm{e}}$ jirongyi@aoe.ac.cn, \\ ${ }^{*}$ corresponding author
}

Keywords: Miss-distance detecting, image rotation, parameter calibration, laser tracker.

Abstract. Laser tracker is an important optical instrument for precise geometric coordinate measurement in large scale. Position sensitive detector (PSD) is one core miss-distance detecting sensor of laser tracker to achieve precise cooperation target tracking. The tracking parameter calibration accuracy based on PSD has remarkably direct influence on the performance of tracking and measurement. Firstly, a laser tracking measurement system is presented, and the working principle of the system's tracking unit based on PSD is introduced. Then the hardware and software design of miss-distance detecting module is described on the basis of PSD according to the actual demand. The experimental result shows that the stability of spot position is better than $\pm 2.0 \mu \mathrm{m}$ in the square effective area $\left(4 \times 4 \mathrm{~mm}^{2}\right)$. Finally, from precise detecting principle and system structure analysis, the forming mechanisms of tracking zero point and phenomena of laser spot rotating with azimuth axis rotation are analysed. Their influence on target tracking is discussed, and two novel calibration methods of tracking zero point and phase angle of laser spot rotating coordinate are proposed. By these calibration methods and precision displacement experiment the relationship model in tracking state between PSD displacement and target miss-distance based on data fitting and interpolation is set up. Experimental results indicate that the detection module design and the tracking parameter calibration method can well meet the demand of the precise and fast target tracking.

\section{Introduction}

Laser tracker is a kind of widely used high-precision optical coordinate measurement instrument. Laser tracker is also known as laser tracking interferometer, just as the word implies, and distance interference measurement is based on the target's precise tracking. To achieve rapid and precise tracking, the servo control system must obtain in real time the high-precision target miss-distance information in two dimensions. Laser spot displacement detecting based on position sensitive detector(PSD) is one important miss-distance sensing method. Compared with four-quadrant detector, PSD has characteristics of more accuracy and no dead zone[3]; in contrast with camera, it has much more fast response and smaller size[4,5]. Hence it becomes one of the core miss-distance measurement elements in the laser tracking system. However, laser tracker is a complex opto-mechatronics integration instrument. Although PSD displacement has one-to-one match with target miss-distance, the relationship varies with opto-mechanical components poses and tracking state. For example, pose and installing location of PSD affects tracking zero point directly, and laser spot on PSD rotates with azimuth tracking motor rotation. These problems attract researcher to investigate effective and convenient calibration means to set up the analytical expression between target miss-distance and PSD displacement. Only by this way the tracking measurement system could achieve good tracking performance. 


\section{Laser tracking measurement principle}

The working principle of laser tracking interferometer system is shown as Fig.1. The light beam emitted from laser is divided into two beams, one of which transfers to the interferometer directly, and the other of which is reflected by tracking mirror and heads to target. After the target reflection, the light beam returns beam splitter through tracking mirror and is divided into two new light beams. One is reflected to interferometer for distance measurement and the other one is reflected to PSD for target miss-distance detecting and tracking. When light beam locates on the centre of target, in theory the PSD unit's output of target miss-distance is zero. Otherwise, the specific coordinate value representing the target deviating from target ball centre is calculated and outputted in real time. At this time the tracking system drives tracking mirror rotating around reflection base point, which reduces miss-distance to zero and realize tracking measurement of the target[3]. Considering the precondition of interferometer continuously outputting right distance is the target miss-distance keeping below several millimetres in the wide distance range between tens of meters and one hundred meters. Hence the tracker's effective field of view is very limited compared with the traditional opto-electronics tracking system, which requires PSD displacement detecting unit has the stable, high accuracy detecting ability and rapid, convenient miss-distance resolving method.

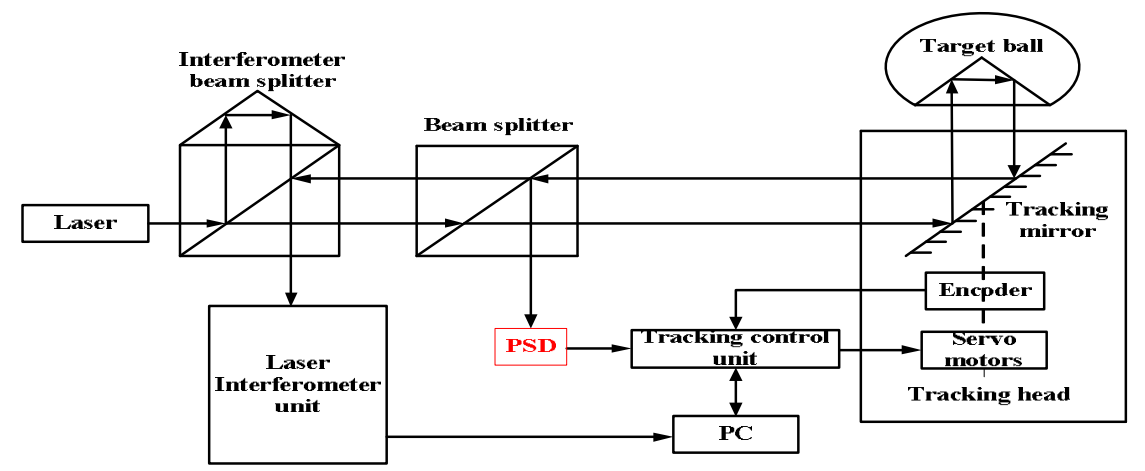

Fig.1 Laser tracking measurement system diagram

\section{System design of miss-distance detecting based on PSD}

Due to the advantages such as small volume, high resolution, fast response, no dead-time etc., Position sensitive detector (PSD) is widely used in precision position detection of coordinates and posture[6-8]. It has gradually become one of the key position feedback elements in the tracking system $[9,10]$. In this paper the two-dimensional PSD selects 2L10_SU7 with fast response $(0.4 \mu \mathrm{s})$ and high-resolution (better than $0.1 \mu \mathrm{m}$ ) provided by Sweden SiTek Company. Its active square area is $10 \times 10 \mathrm{~mm} 2$; position non-linearity is within $\pm 0.8 \%$; typical value of reverse voltage is $15 \mathrm{~V}$.

\section{Hardware design of detecting unit}

Target miss-distance detecting system based on PSD mainly includes PSD and its signal conditioning circuit, AD data acquisition module, data processing and communication unit etc. System's block diagram is shown in Fig. 2. Firstly optical signal from laser is incident on PSD's active area through special optical path in laser tracker. It prompts PSD generating four current signals. Through I/V conversion, level shift and amplification, the four current signals are converted to proper voltage signals in the range from 0 to $10 \mathrm{~V}$ or $-10 \mathrm{~V}$ to 0 ; after $\mathrm{AD}$ module's synchronous parallel sampling and analogy-digital conversion, the voltage signals are sent to data processing unit based on FPGA, where the digital signals are conducted logic operation and digital filtering. Finally the spot position result is transferred to tracking control unit in the form of low voltage differential signal(LVDS).

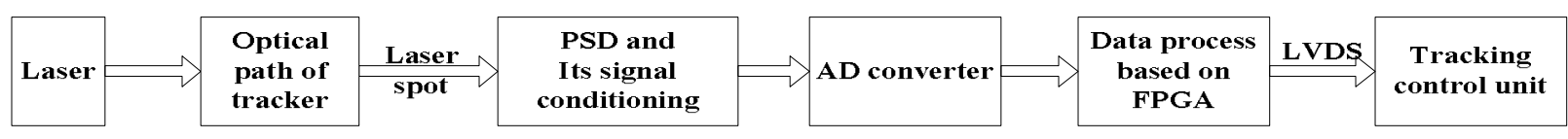

Fig. 2 Overall concept of miss-distance detecting unit 


\section{Software design and experimental results}

Based on Spartan-3E series FPGA supplied by Xilinx, the system software are top-down designed and modularly programmed. AD conversion process, filtering algorithm and data processing method on spot centre coordinates is proposed and developed, and Verilog HDL and Melay finite state machines are applied. The program flow chart is shown as Fig. 3.

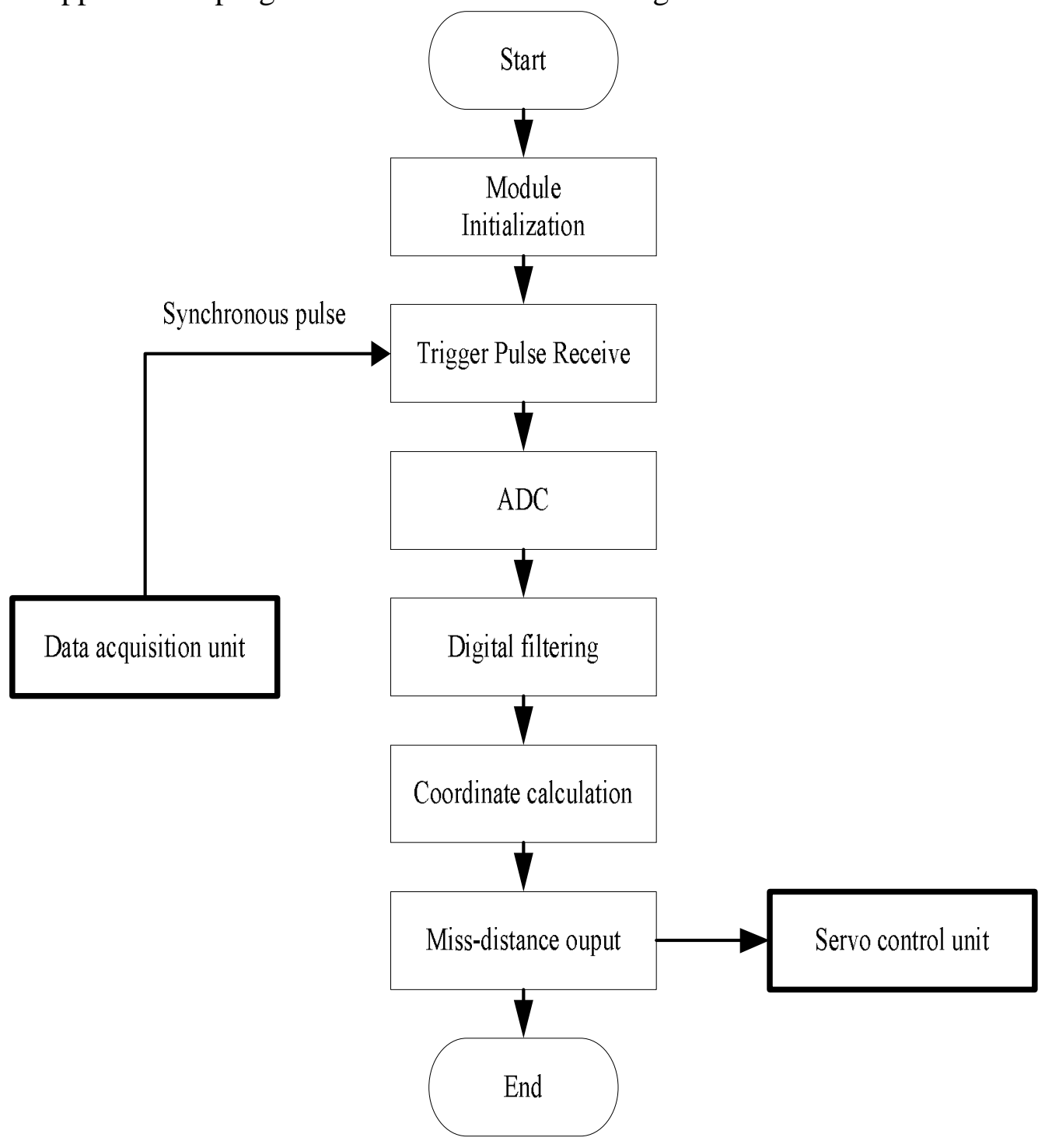

Fig. 3 Flow chart of Program

In the laser tracking system, considering the divergence angle and interference characteristic, the laser wavelength is chosen as $632.8 \mathrm{~nm}$. In the detecting process, the detecting unit is interfering inevitably by the complicated internal laser source and devices inside the tracker, power supply as well as motor driving current. To examine the effectiveness of the hardware and test the stability of measurement results, the experiment is carried out based on precise two-dimensional displacement platform. The results are shown as follows. 


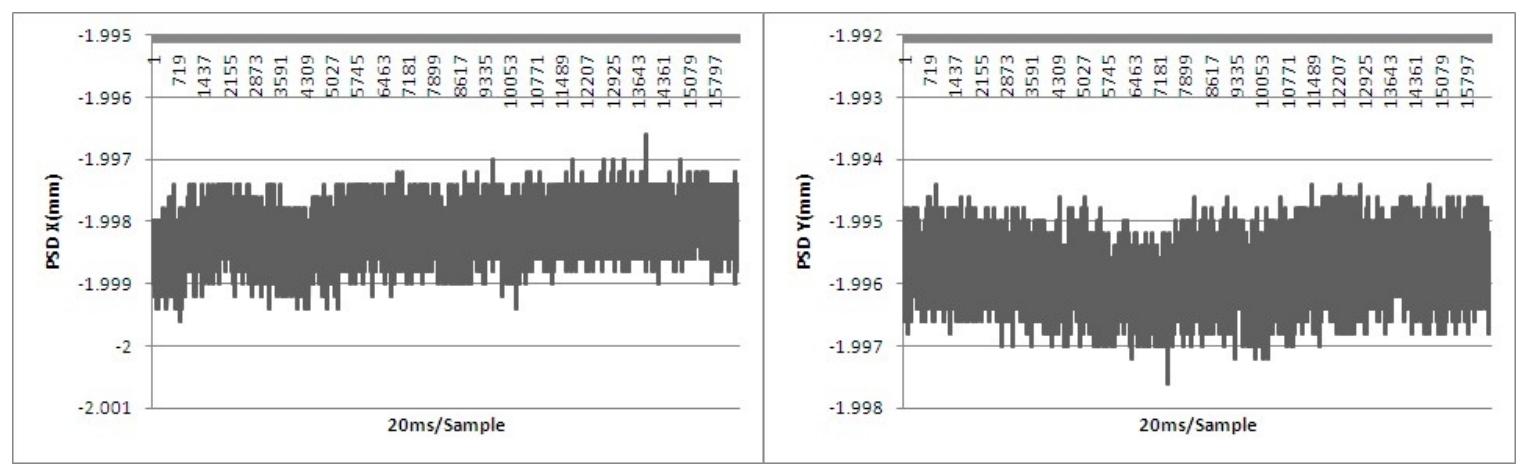

(a) Displacement fluctuation when light spot is around $(-2,-2)$
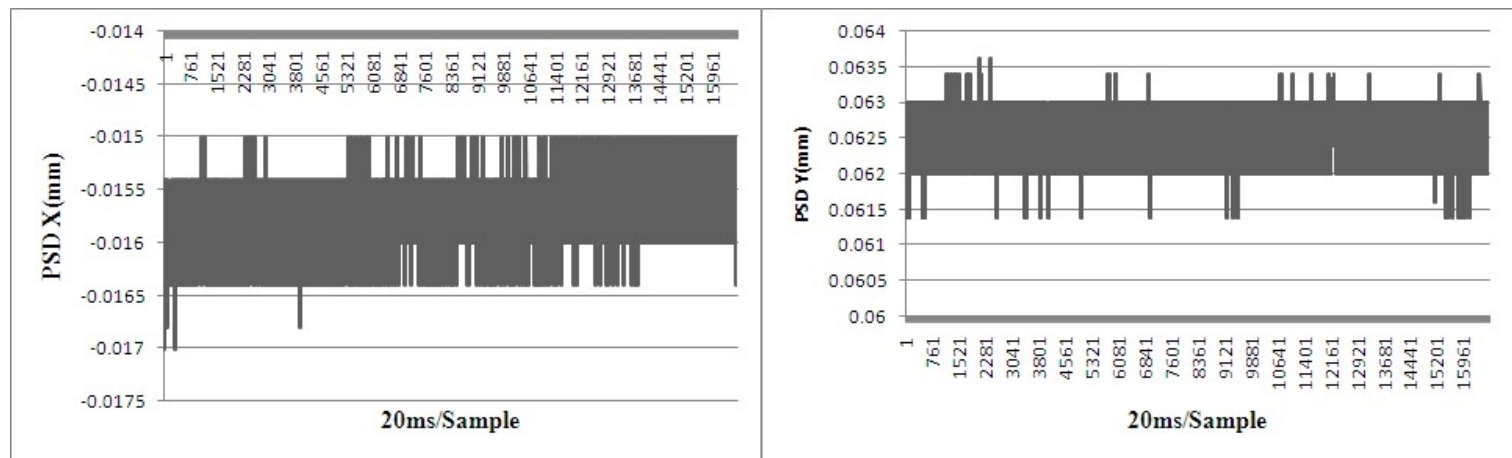

(b) Displacement fluctuation when light spot is around $(0,0)$
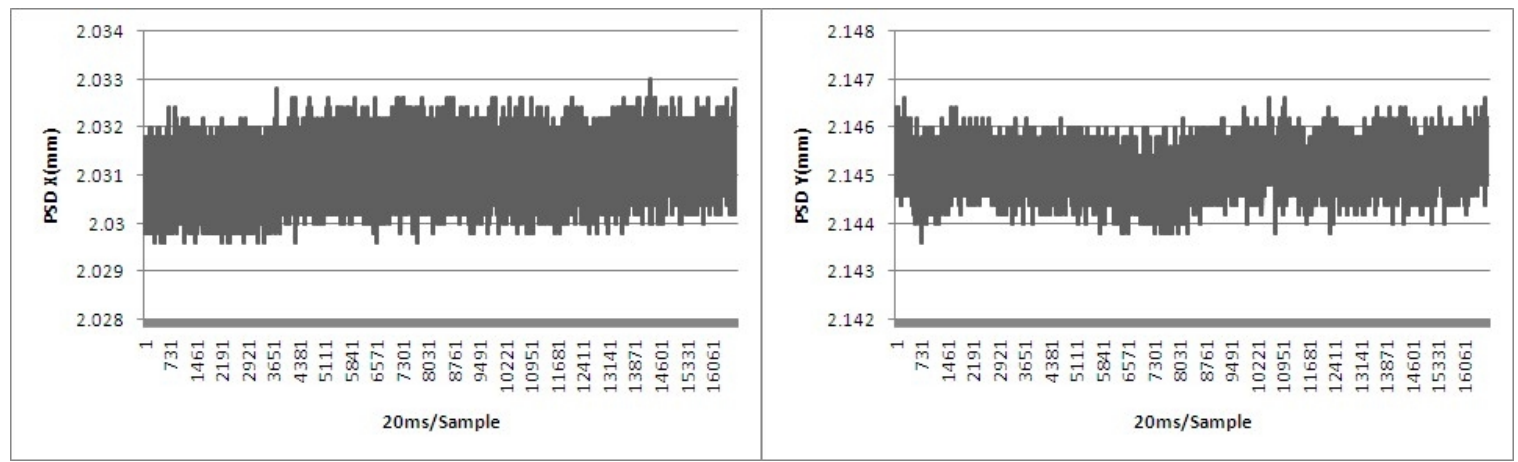

(c) Displacement fluctuation when light spot is around $(2,2)$

Fig.4 The experimental result of PSD displacement

From the Fig.4(a), it can been that the differences of peak value from bottom value of PSD X and PSD Y fluctuation are $3.0 \mu \mathrm{m}$ and $3.2 \mu \mathrm{m}$ respectively; and from Fig.4(c), the differences are $3.4 \mu \mathrm{m}$ and $3.0 \mu \mathrm{m}$. While Fig.4(b) showed that the differences are no more than $2.0 \mu \mathrm{m}$. This is caused by the PSD's inherent PIN structure and inhomogeneous distribution of P-type layer resistivity [11,12], that is, so called PSD bordering effect (when the spot goes on bordering, the detecting accuracy decrease gradually). Limit to the length of the paper, only the result of the above typical point in square detecting area of $x \in(-2,2), y \in(-2,2)$ is presented. Actually, the stability is better than $\pm 2 \mu \mathrm{m}$ in the whole square area.

\section{Calibration of tracking Parameters}

The experimental results obtained from the above software and hardware solutions show the high detecting stability of the PSD unit. On the basis of it, the analytical expression of target motion and spot displacement in tracking state should be established in order to achieve tracking for the cooperative target. Before the establishment, the laser tracking zero point and spot rotating parameter need firstly to precise calibrate. 


\section{Calibration method of tracking zero point}

In the installation process, the PSD posture and optical path adjustment is usually not to be able to reach the ideal state, therefore the geometric centre of the PSD may differ from the tracking zero point (incoming laser spot reflecting from target ball centre and the tracking mirror). In fact the aim of the laser tracker's control system is to drive the two-dimensional turntable rotation and make the target miss distance to be 0 . Therefore, which point is the actual tracking zero point corresponding to the 0 miss-distance value is very important. The accuracy of the tracking zero point calibration does not only influence the tracking performance but also system measurement precision. In this paper a novel calibration method is proposed without the aid of any external devices. Firstly, adjust the tracking mirror downward (nearly vertical), it can be seen that the spot moving trajectory forming like a circle varies with the mirror rotating one round in azimuth. As the emerging laser beam is coaxial with azimuth rotating axis (The geometry position of laser tracker's mechanical structure has previously been precision alignment), if the tracking mirror is not perpendicular to the incoming beam, then there is a small intersection angle between the incoming and the emerging ones. It leads to the circular trajectory, which is formed by the spot movement. The diameter of the trajectory circle increase with the intersection angle becoming bigger, and vice versa. In particularly, the laser spot doesn't change with the mirror rotation driven by the azimuth rotating axis. In the calibration experiment process, adjusting the tracking mirror vertical angle continuously and driving the azimuth rotating axis until the intersection angle is small enough and the diameter of the trajectory circle is less than $0.2 \mathrm{~mm}$. The centre of the circle is defined as theoretical tracking zero point of the PSD. The calibration schematic of tracking zero point is as Fig.5.

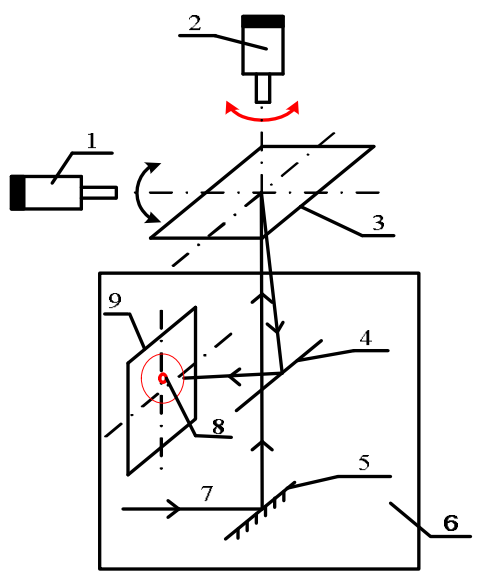

1: Elevation rotation motor, 2: Azimuth rotation motor, 3: tracking mirror, 4: beam splitter,

5: reflector, 6: tracker body, 7: laser, 8: tracking zero point, 9: PSD

Fig.5 Calibration schematic of PSD Tracking zero point

In order to improve calibration accuracy, the centre can be obtained using average method from many time experiments. Usually the differences of circle centre coordinates are not more than $3 \mu \mathrm{m}$. The experimental results are shown as Fig. 6. According to the numeric calculation, the tracking zero point is $(-0.022 \mathrm{~mm},-0.088 \mathrm{~mm})$.

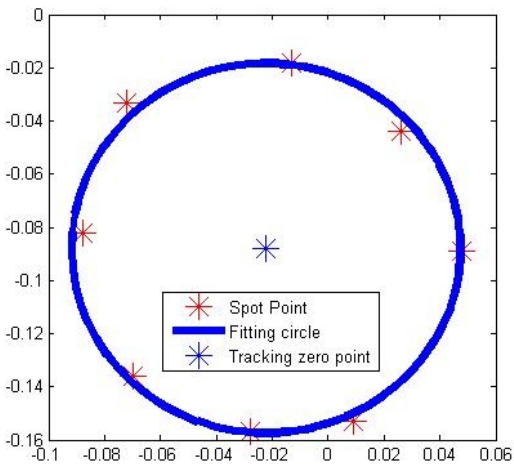

Fig.6 the experimental results of spot movement trajectory 


\section{Phase angle calibration of spot rotation}

Laser tracking control process is driving the turntable to reduce the target miss-distance to zero, however as the stated above the spot position (displacement) corresponding to the target miss-distance rotates with the tracking mirror azimuth rotation, hence the spot position (displacement) must be converted to the miss-distance in the rotating coordinate. Then based on the new displacement, the control system can achieve effective target tracking. Obviously the rotating coordinate has its own absolute initial angle (phase angle), which needs to be calibrated in advance.

Suppose the square PSD itself constituting a two dimensional coordinate system (X, Y)and the extension of the light spot orthogonal movement trajectory forming a coordinate system $\left(X^{\prime}, Y^{\prime}\right)$. When the azimuth rotating motor keeps a certain angle $\alpha$, and the condition is satisfied as that both $\mathrm{X}$ with $\mathrm{X}^{\prime}$ and Ywith $\mathrm{Y}^{\prime}$ are parallel and remain in the same direction, then the absolute angle $\alpha$ is defined as the phase angle of the rotating coordinate $\left(\mathrm{X}^{\prime}, \mathrm{Y}^{\prime}\right)$. The principle of the spot rotation phenomenon is shown in fig. 10 .
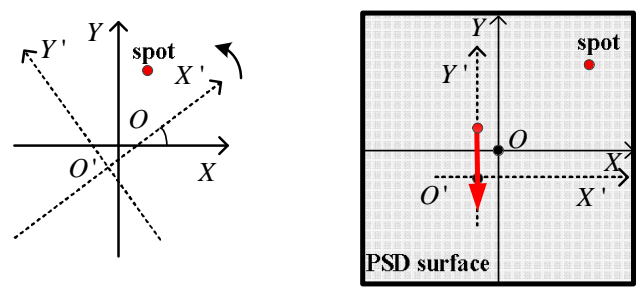

Fig.7 Schematic of spot rotation with coordinate and initial angle calibration concept

In this situation, fixing the target in the same height with laser tracker's elevation rotating axis and driving the mirror to make the laser beam scan the target ball and cross the ball centre, the spot in the PSD move in line $\mathrm{Y}^{\prime}$ direction and $\mathrm{x}$ coordinate value remain nearly the same except in PSD bordering. The experimental result of PSD coordinate is showed as Fig.8. According to this experiment, the actual initial angle is $78.1143^{\circ}$.

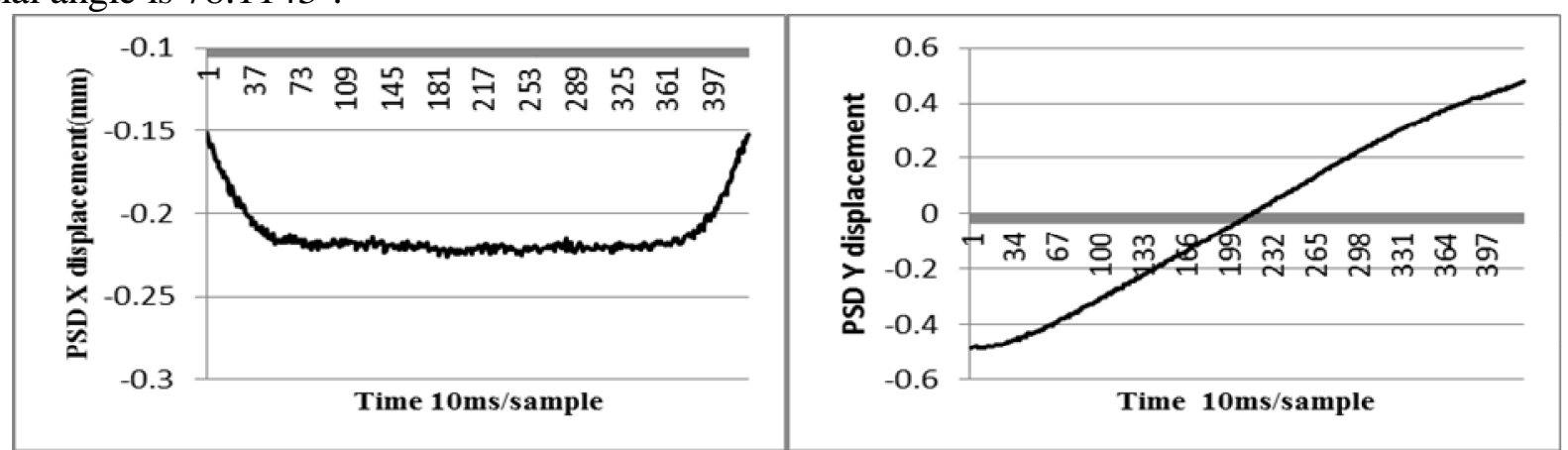

Fig.8The PSD coordinate values when the laser beam scan the target ball at the initial angle

\section{Calibration of relationship between miss-distance and displacement}

Since the laser beam expands and shrinks by optical path system and PSD installation is not in ideal state, the analytical relationship between PSD displacement and target miss-distance is not strict linearity with ratio of 1:1, so the quantitative relationship between the displacement and miss-distance need to be calibrated in advance to ensure good tracking performance. Before the experiment, firstly driving the servo motor to keep the mirror as the phase angle and adjusting the target ball centre to be at the same height with the middle mirror. Secondly moving the target in horizontal and vertical direction respectively, make sure that laser spot cross the tracking zero point and recording the coordinate $\mathrm{x}$ and $\mathrm{y}$ values. The experimental scheme is showed as figure 9. 


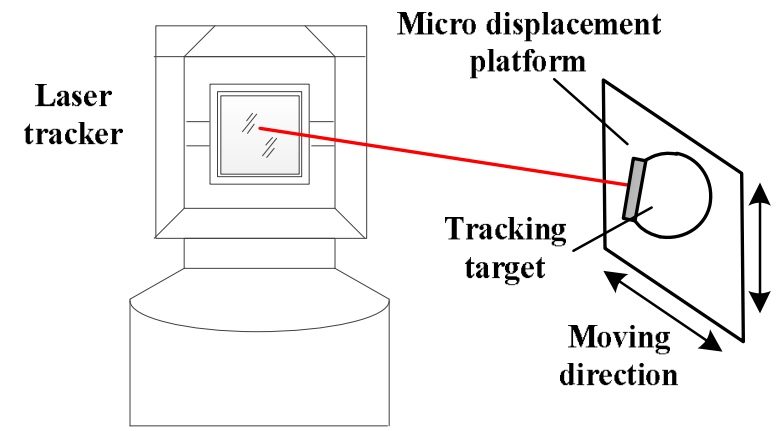

Fig.9 Schematic of relationship calibration between miss-distance and PSD displacement As the target moves horizontally and vertically, the relationship between the movement distance and the laser spot displacement on the PSD is shown as Fig.10. To estimate scale factor, the least squares fitting algorithm is adopted. The experimental result is shown as Fig. 10.

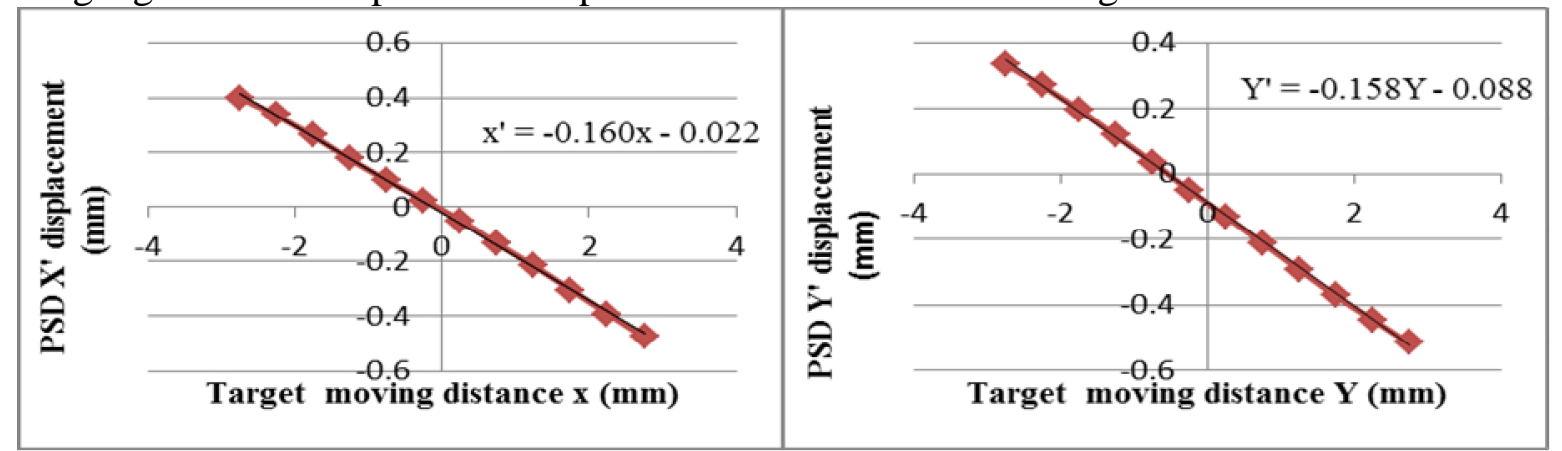

Fig.10 The result of linear relationship calibration between displacement and miss-distance

According to the calibration result of tracking zero point, phase angle and linear ratio, as the azimuth angle is $\theta$, the target miss distance calculation equation in tracking state can be expressed as:

$$
\left\{\begin{array}{l}
X=-\frac{1}{0.160}\left(\left(X^{\prime}+0.022\right) \cos (\theta-78.1143)+\left(Y^{\prime}+0.088\right) \sin (\theta-78.1143)\right) \\
Y=-\frac{1}{0.158}\left(\left(Y^{\prime}+0.088\right) \cos (\theta-78.1143)-\left(X^{\prime}+0.022\right) \sin (\theta-78.1143)\right)
\end{array}\right.
$$

\section{Summary}

The working principle of the laser tracker is introduced and the important role of improving tracker's tracking performance by precise target miss-distance detecting is analysed. Based on PSD, the miss-distance hardware and software solutions is described. The experimental result indicates the spot displacement varies less than $\pm 2 \mu \mathrm{m}$ in the central square detecting zone ( $4 \mathrm{~mm} \times 4 \mathrm{~mm})$, and is more stable and precise than the Four-quadrant detector. Hence PSD could satisfy the tracker demand better. To improve laser tracking performance the systematically calibration methods are researched. A novel tracking zero point calibration method is presented in detail; following the proposed method, the coordinate error is less than $3 \mu \mathrm{m}$. Similarly the phase angle of rotating coordinate calibration method is introduced and the angle error is less than $0.1^{\circ}$. Then the precision displacement experiment is carried out. At last the quantitative relationship between miss-distance and PSD displacement setting up method in tracking state is also discussed and presented in detail. On the basis of the above calibration method, the relationship expression was applied successfully in the laser tracking system and achieved satisfactory performance.

\section{References}

[1] ZHOU Pei-song, LU Rong-sheng, ZHOU Wei-hu, et al. Measurement System for Laser Tracker's Target-missing Quantity Based on Four-quadrant Detector[J]. ELECTRO-OPTIC TECHNOLOGY APPLICATION,2014,29(1):75-79 
[2] SU Yan-zhao, Li Ai-hua, Wang Tao, et al. Visual tracking of moving objects based on piecewise fusion weight and multi-strategy[J]. Optics and Precision Engineering, 2015, 22(12): 3409-3418.

[3] Song Lin, Cheng Yongmei, Liu Nan, Liu Xialei. Algorithm of vision tracking for UAV navigation based on multi-constraint KLT[J]. Infrared and Laser Engineering,2013, 42(10): 2828-2835.

[4] LIU Jiao-yue, YANG Ju-qing, DONG Deng-feng, ZHOU Wei-hu. Optoelectronic Aiming and Orientation System of Laser Tracker[J]. Optics and Precision Engineering, 2015, 23(6):1558-1564.

[5] Song Dianyou , Liu Tiegen, Ding Xiaokun, et al. Precise PSD Micro-Displacement Online Measurement System[J]. Journal of Tianjin University(Science and Technology), 2013, 46(2):168-173

[6] Ying Duan, Lan Qin, LianXue, et al. Multi-beam synchronous measurement based on PSD phase detection using frequency-domain multiplexing[C]. 2013 Proc. SPIE 8916, Sixth International Symposium on Precision Mechanical Measurements, vol.8916: 89163C.

[7] ZHANG Ya-juan, QIU Zu-rong, LI Xing-hua, et al. Laser tracking coordinate measurement system based on PSD[J]. Transducer and Micro system Technologies, 2011, 30(7): 116-119.

[8] J. Conte, J. Santolaria, A.C. Majarena, et al. Identification and kinematic calculation of Laser Tracker errors[C]. The Manufacturing Engineering Society International Conference, 2013, 63: 379-387.

[9] Zhang Min, Liang Yanbing. Experiment on two dimensional quadrilateral position sensing detector[J].Infrared and Laser Engineering, 2013, 42(2):459-464.

[10] Toyomi Fujita. New types of semiconductor image position sensitive device[J]. Optical Engineering, 2009, 48(9): 1-5. 